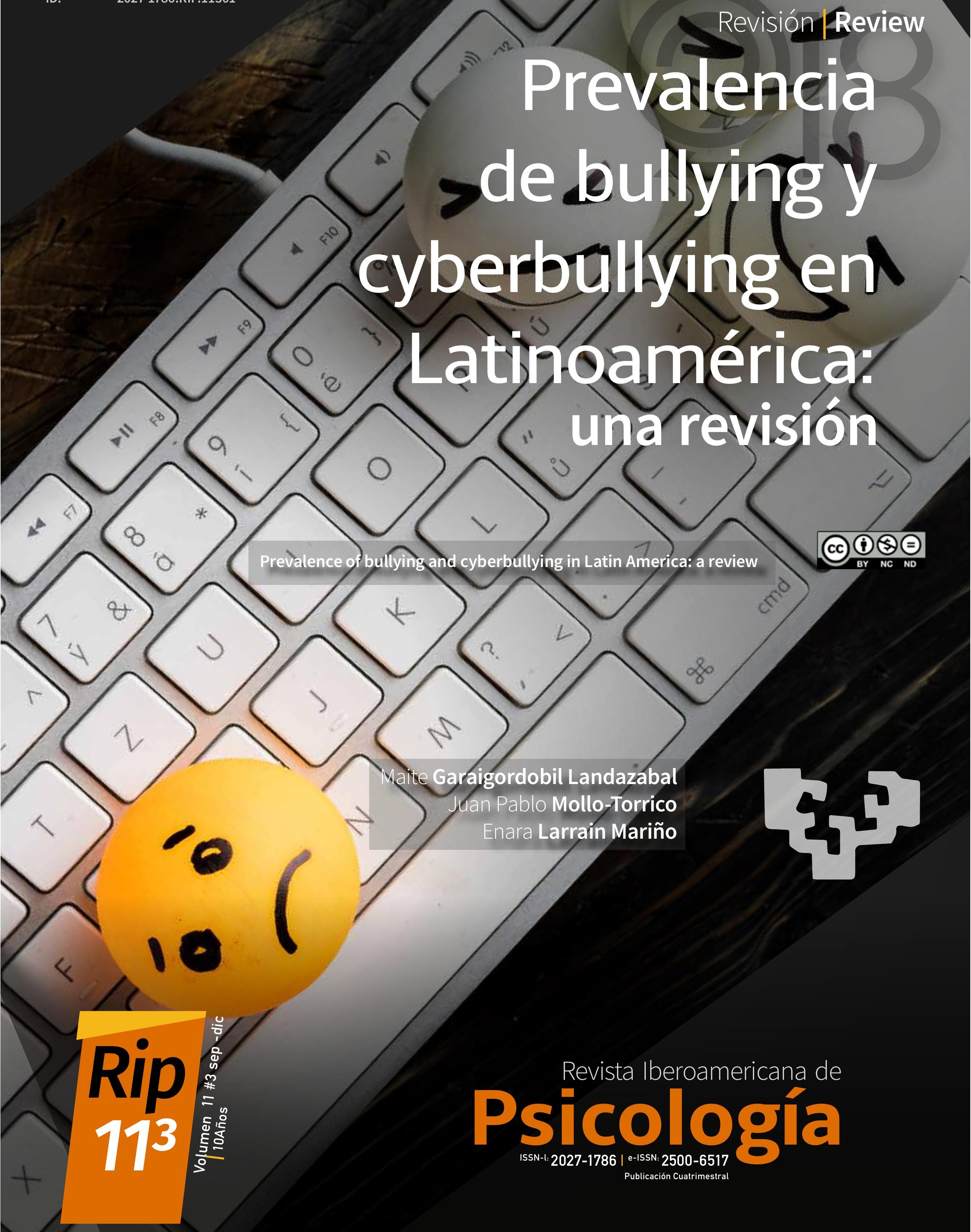


Revista Iberoamericana de

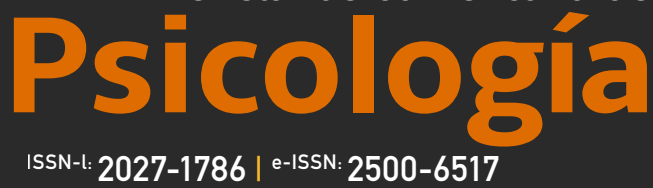
Publicación Cuatrimestral

ID:

2027-1786.RIP.11301

Title: $\quad$ Prevalence of bullying and

cyberbullying in Latin America: a review

Título: Prevalencia de bullying y cyberbullying en Latinoamérica: una revisión

Alt Title / Título alternativo:

[en]: Prevalence of bullying and cyberbullying in Latin America: a review

[es]: Prevalencia de bullying y cyberbullying en Latinoamérica: una revisión

\section{Author (s) / Autor (es):}

Garaigordobil Landazabal,Mollo-Torrico, Larrain Mariño.

Keywords / Palabras Clave:

[en]: bullying, cyberbullying, prevalence, Latin America, systematic review

[es]: bullying, cyberbullying, prevalencia, Latinoamérica, revisión sistemática

\section{Proyecto / Project:}

Estudio financiado por la Universidad del País Vasco (UPV/EHU) (PPG17/31), y por la Red PROEM "Promoción de la salud mental emocional en adolescentes", Ministerio de Ciencia, Innovación y Universidades (PSI201790650-REDT).

Financiación / Funding:

Estudio financiado por la Universidad del País Vasco (UPV/EHU) (PPG17/31), y por la Red PROEM "Promoción de la salud mental emocional en adolescentes", Ministerio de Ciencia, Innovación y Universidades (PSI2017-90650-REDT).

Submited: 2018-08-21

Dra Maite Garaigordobil Landazabal, MSc Psi sp

\section{AutorlD: $\quad$ ID: 55973780400 \\ Research ID: C-8969-2011}

ORCID: $\quad$ 0000-0002-8621-6245

Source |Filiacion:

Universidad del Pais Vasco

$\mathrm{BIO}$

Doctora en Psicología, Especialista en Psicología Clínica, y Catedrática de Evaluación y Diagnóstico Psicológicos en la Facultad de Psicología de la Universidad del País Vasco. Docente e investigadora en temas relacionados con la intervención psicológica en contextos clínicos y educativos City | Ciudad:

San Sebastián-Donostia [es]

e-mail:

maite.garaigordobil@ehu.eus

\section{Resumen}

El objetivo de este trabajo consiste en realizar una revisión sistemática de las investigaciones que aportan porcentajes de víctimas y agresores de bullying y cyberbullying en Latinoamérica (2005-2018). Con esta finalidad se utilizan las principales bases de datos

(Scopus, WebOfScience, ERIC). Se han

encontrado 51 estudios, 35 sobre bullying,

10 de cyberbullying y 6 aportan información de ambas modalidades de acoso. Los resultados evidencian una alta prevalencia de bullying y, aunque en menor medida también de cyberbullying, en todos los países de

Latinoamérica donde el fenómeno ha sido estudiado (Colombia, México, Argentina, Brasil,

Bolivia, Perú, Chile, Nicaragua, Venezuela,

Panamá, Ecuador, y Puerto Rico). La revisión

confirma una significativa prevalencia de

bullying ocasional/frecuente (víctimas 4.6\%50\%; agresores 4\%-34.9\%) y de cyberbullying ocasional/frecuente (cibervíctimas 3.5\%-17.5\%; ciberagresores 2.5\%-58\%). La mayoría de los implicados son varones. El tipo de acoso más frecuente es el verbal, seguido del psicológico

y el físico. Aunque el bullying cara-a-cara, especialmente la violencia física, disminuye con la edad, el cyberbullying sigue presente en

la adolescencia tardía y la juventud. Entre las

conductas de cyberbullying más frecuentes identificadas en esta revisión caben destacar:

envío de mensajes desagradables, insultos,

amenazas, realizar comentarios y hacer circular rumores para desprestigiar o ridiculizar

a la víctima, difundir fotos y vídeos ofensivos para la víctima, robo de la contraseña. .

La magnitud de los datos epidemiológicos hallados en esta revisión enfatiza la necesidad

de seguir investigando el tema, además de implementar programas de prevención/ intervención del bullying/cyberbullying, durante la infancia, la adolescencia y la juventud.

\section{Citar como:}

Garaigordobil Landazabal, M., Mollo-Torrico, J. P., \& Larrain Mariño, E., (2018). Prevalencia de bullying y cyberbullying en Latinoamérica:: una revisión. Revista Iberoamericana de Psicología issn-l:2027-1786, 11 (3), 1-18. Obtenido de: https://revistas.iberoamericana.edu.co/index.php/ripsicologia/article/view/1435-4972

Juan Pablo Mollo-Torrico, [Dr] MSc PS

Source | Filiacion:
Universidad del Pais Vasco

BIO:

Licenciatura en Psicología (Universidad Mayor de San Simón, Bolivia). Master en Psicología: Individuo, Grupo, Organización y Cultura Universidad del País vasco. En proceso de realización de tesis doctoral

City | Ciudad:

San Sebastián-Donostia [es]
Abstract

The aim of this work was to carry out

a systematic review of the researches

that provide percentages of victims and perpetrators of bullying and cyberbullying in Latin America (2005-2018). For this purpose the following main databases were used: Scopus; WebOfScience; ERICScopus; WebOfScience; and ERIC. A total of 51 studies were found: 35 on bullying, 10 on cyberbullying, and 6 that provide information about both types

of harassment. The results show a high prevalence of bullying and also cyberbullying (to a lesser extent) in all the countries of Latin

America where the phenomenon has been studied (Colombia; Mexico; Argentina; Brazil;

Bolivia; Peru; Chile; Nicaragua; Venezuela;

Panama; Ecuador; and Puerto Rico). Such

systematic review confirms a significant prevalence of occasional/frequent bullying (4.6\% - 50\% victims; 4\%-34.9\% bullies) and of occasional/frequent cyberbullying (3.5\% 17.5\% cybervictims; $2.5 \%$ - 58\% cyberbullies). Most of those involved are males. The most

frequent type of bullying is verbal, followed psychological and physical bullying. Although face-to-face bullying (especially physical violence) decreases with age, cyberbullying

is still present in late adolescence and youth. Among the noteworthy cyberbullying behaviors identified in this review are: sending nasty messages; insults; threats; making comments or spreading rumors to discredit or ridicule the victim; disseminating pictures and videos that are offensive to the victim; password theft; among others.

The magnitude of the epidemiological data

found in this review emphasizes the need to further investigate the issue, in addition

to implementing bullying/cyberbullying prevention/intervention programs during childhood, adolescence and youth. Enara Larrain Mariño, [Dr] MSc Enf

Source | Filiacion:

Universidad del Pais Vasco

$\mathrm{BIO}$

Graduada en Enfermería. Máster de Investigación en Psicología: Individuo, Grupo, Organización y Cultura. Personal Investigador en Formación becada por el Gobierno Vasco (PRE_2017_1_0017) para la realización de tesis doctoral

City | Ciudad:

San Sebastián-Donostia [es] 


\title{
Prevalencia de bullying y cyberbullying en Latinoamérica:
}

\author{
una revisión
}

\author{
Prevalence of bullying and cyberbullying in Latin America: a review
}

\author{
Maite Garaigordobil Landazabal \\ Juan Pablo Mollo-Torrico \\ Enara Larrain Mariño
}

\section{Introducción}

La definición más aceptada y utilizada de bullying es la formulada por Olweus (1973; 1993; 2013). Este investigador considera que un estudiante está siendo intimidado cuando otro estudiante o grupo de estudiantes le dice cosas mezquinas o desagradables, se ríe de él o ella o le llama por nombres molestos o hirientes, cuando se le ignora completamente, se le excluye de su grupo de amigos o se le retira de actividades a propósito, cuando se le golpea, patea, empuja o amenaza, cuando se le cuenta mentiras o falsos rumores sobre él o ella, se le envía notas hirientes y se trata de convencer a los demás para que no se relacionen con él o ella. Estas conductas ocurren frecuentemente y es difícil para el estudiante que está siendo intimidado defenderse por sí mismo.

En los últimos años ha surgido una nueva forma de bullying denominada cyberbullying, que consiste en utilizar las Tecnologías de la Información y la Comunicación, principalmente Internet (correo electrónico, mensajería instantánea o "chat", páginas web o blogs, videojuegos online...), y el teléfono móvil, para ejercer el acoso psicológico entre iguales. Las vías utilizadas para ejercer cyberbullying son variadas: mensajes de texto (SMS), acoso telefónico (llamadas anónimas, amenazantes o insultantes al móvil...), grabaciones de agresiones físicas o vejaciones que son difundidas vía móvil o en Internet, acoso a través de fotografías que se difunden por los móviles o se suben a YouTube, correos electrónicos, mensajería instantánea, en sesiones de chat, en las redes sociales (Facebook, Whatsapp, Twitter, Instagram. ..), páginas web (blogs, fotologs...) (Garaigordobil, 2018).

La preocupación social por el bullying y el cyberbullying se ha incrementado. Progresivamente, la sociedad ha tomado conciencia de la gravedad que tiene la violencia entre iguales, tras haberse constatado que el bullying/cyberbullying tienen consecuencias muy negativas para todos los implicados. Aunque las consecuencias más graves se evidencian en las víctimas (ansiedad, depresión, estrés postraumático, ideación suicida, suicidio, trastornos en el sueño, en la alimentación, disminución del rendimiento académico...), también ser agresor está asociado con bajo rendimiento académico, con consumo de alcohol y drogas, con el desarrollo de una personalidad sin empatía, cruel e insensible frente al dolor ajeno, con muchas conductas antisociales, con dificultades para cumplir las normas, para controlar la ira y la impulsividad, todo ello generador de numerosos problemas de adaptación personal, social y laboral a lo largo de su vida (Garaigordobil, 2018). Además, estas consecuencias en muchas ocasiones se extienden a corto y largo plazo en la vida de las víctimas y los agresores. Por ello, en los últimos años se está produciendo un incremento de los estudios que analizan el bullying en todas sus modalidades, identificando su prevalencia en todos los países del mundo, explorando factores de protección y de riesgo, elaborando instrumentos de evaluación y propuestas de prevención e intervención. .

Transcurridos más de $\mathbf{4 0}$ años desde los pioneros estudios epidemiológicos de bullying cara-a-cara de Olweus (1973), y de cyberbullying de Finkelhor, Mitchell \& Wolak (2000), se han desarrollado numerosos estudios de prevalencia del bullying/cyberbullying en el mundo. Una revisión reciente de $\mathbf{3 0 9}$ investigaciones de prevalencia (Garaigordobil, 2017b) ha encontrado en estos estudios una horquilla entre 2 y 16\% de bullying y entre $\mathbf{1}$ y $\mathbf{1 0} \%$ de cyberbullying severos, lo que permite afirmar que bullying y cyberbullying son fenómenos que se producen en todos los países y en todos los niveles socio-económico-culturales. Además, por lo que sabemos de sus consecuencias, también se puede concluir que es un problema de salud pública. La significativa prevalencia y las graves consecuencias hacen relevante identificar su prevalencia en todos los países, para después implementar propuestas para prevenir y protocolos para intervenir cuando la victimización se produce. 
El trabajo planteado forma parte de una línea de investigación sobre bullying/cyberbullying que se inició en 2003, y se ha configurado con sucesivos trabajos (Garaigordobil, 2013; 2015; 2017a; 2017b); (Garaigordobil \& Oñederra, 2008; 2009; 2010); (Garaigordobil \& Martínez-Valderrey, 2018); (Machimbarrena \& Garaigordobil, 2017). Este estudio tiene como principal objetivo llevar a cabo una revisión sistemática de las investigaciones que han explorado la prevalencia de bullying/ cyberbullying en los países Latinoamericanos. Esta revisión se realiza porque no existen revisiones de la prevalencia de este fenómeno que permitan valorar el porcentaje de estudiantes que sufre estas conductas en los distintos países de Latinoamérica, y además permitirá identificar la cantidad de estudios de prevalencia realizados en los países Latinoamericanos.

\section{Método}

En este estudio se ha utilizado un diseño de investigación de observación en retrospectiva, en el cual se han añadido aquellos artículos que analizan la prevalencia de bullying y cyberbullying en los países de Latinoamérica, desde $\mathbf{2 0 0 5}$ hasta la actualidad. Con el objetivo de identificar y examinar los estudios de investigación relevantes de manera sistemática, la búsqueda se ha realizado en las siguientes bases de datos: Scopus, WebOfScience y ERIC. Para la revisión se han utilizado los siguientes términos de búsqueda: bullying, cyberbullying, Latinoamérica y prevalencia en título, resumen o palabras clave.

Con relación a los criterios de inclusión y exclusión, se han seleccionado aquellos artículos cuyo objetivo principal es aportar datos de la prevalencia del bullying/cyberbullying en todos los países de Latinoamérica, excluyendo otros trabajos que aunque contienen algunos datos de prevalencia, principalmente se centran en evaluar variables relacionadas con el bullying/cyberbullying. Además, se tuvieron en cuenta únicamente los artículos que hablaban del acoso escolar y no de la violencia en general, ya que muchas investigaciones de Latinoamérica analizan la prevalencia de diferentes tipos de violencia entre los adolescentes. Por último, se le ha dado gran importancia a la metodología empleada en los estudios, excluyendo aquellas investigaciones que no reflejan el método de forma precisa y metodológicamente rigurosa. El proceso de selección de los artículos se presenta en el gráfico 1.

Gráfico 1. Diagrama de la selección de artículos realizada

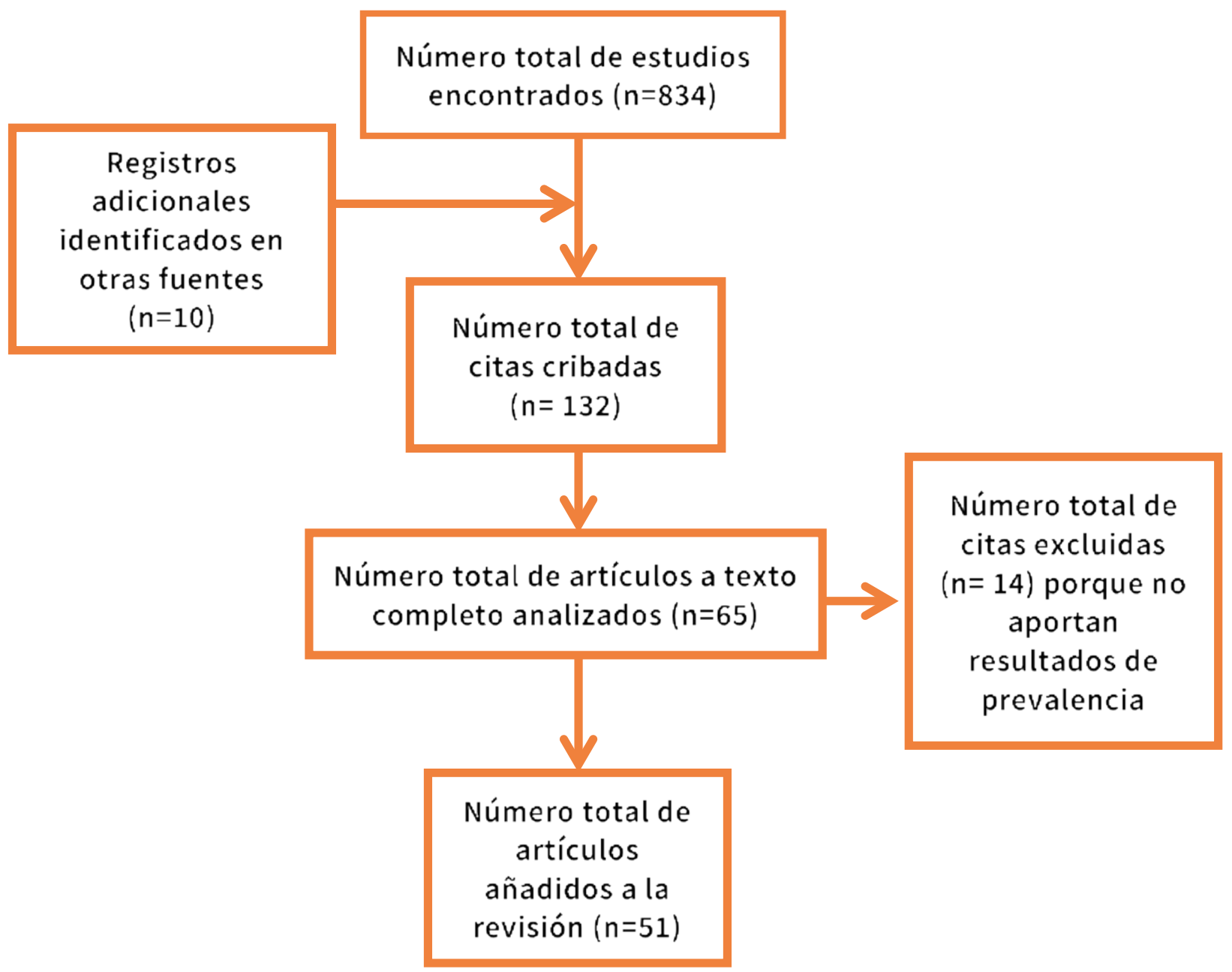


Resultados

En la búsqueda llevada a cabo se han encontrado un total de 51 artículos (ver Tabla 1). De ellos, 35 estudios analizan la prevalencia del bullying, 10 muestran datos sobre el cyberbullying y 6 aportan información tanto de bullying como de cyberbullying. Las muestras de estos estudios llevados a cabo en países de Latinoamérica incluyen niños, adolescentes, jóvenes, y ocasionalmente adultos (profesores, padres, etc.) que aportan información sobre el fenómeno desde lo que cada uno observa. Los resultados de estos 51 estudios sobre la prevalencia de bullying y cyberbullying en Latinoamérica (2005-2018) se pueden observar en la Tabla 1.

Tabla 1. Estudios que exploran la prevalencia de bullying y cyberbullying en Latinoamérica

\begin{tabular}{|c|c|c|c|c|}
\hline Autores (Año) & $\begin{array}{l}\text { Lugar, muestra, } \\
\text { grado y/o (edad) }\end{array}$ & Objetivos de estudio & Resultados bullying & $\begin{array}{c}\text { Resultados } \\
\text { cyberbullying }\end{array}$ \\
\hline Abramovay (2005) & $\begin{array}{l}\text { Brasil } \\
\mathrm{N}=10.069 \\
\text { (población } \\
1.685 .411 \text { ) } \\
\text { enseñanza básica y } \\
\text { media }\end{array}$ & $\begin{array}{l}\text { Analizar casos concretos } \\
\text { de violencias en la } \\
\text { escuela por medio del } \\
\text { relato de alumnos, } \\
\text { profesores y otros que } \\
\text { conviven en la escuela. }\end{array}$ & $\begin{array}{l}4.8 \% \text { víctimas de agresión física; } 38.2 \% \text { ha } \\
\text { sufrido robos; } 83.4 \% \text { percibe violencia en } \\
\text { la escuela. }\end{array}$ & \\
\hline
\end{tabular}

Ortega, Sánchez, Nicaragua, Ortega-Rivera, Del Managua Rey y Genebat $\mathrm{N}=3.042$ (2005) (8-22 años)
Evaluar la prevalencia de acoso escolar en Nicaragua.
Víctimas: entre $25 \%$ y $50 \%$ en función del tipo de agresión, una o más veces: $48.3 \%$ robos; agresión sexual: $4.8 \% ; 45.3 \%$ insultos; $37.5 \%$ agresión física; $37.2 \%$ exclusión; $25.5 \%$ amenazas.

Frecuentemente: $28.9 \%$ robos; agresión sexual: $2.2 \% ; 21.8 \%$ insultos; $20.2 \%$ agresión física; $16.8 \%$ exclusión; $12.2 \%$ amenazas.
Argentina (Central, Patagonia, NOA, Ciudad de Buenos

Kornblit y Adaszko Aires, Cuyo, (2007) Conurbano Bonaerense, $y$ NEA) $\mathrm{N}=4.971$ (15-19 años)
Víctimas: $16.9 \%$ víctimas de violencia; $52.9 \%$ víctimas de hostigamiento.

Evaluar la prevalencia Agresores: $16.6 \%$ de violencia; $51.8 \%$ de de bullying en Argentina. hostigamiento.

Observadores: $34.4 \%$ de violencia; $75.5 \%$

de hostigamiento

Cepeda-Cuervo Pacheco-Durán, García-Barco y Piraquive-Peña (2008)

Colombia, Bogotá

$\mathrm{N}=3.226$

$6^{\circ}$ a $11^{\circ}$

(10-20 años)
Determinar las características y el nivel de acoso escolar en colegios de Bolívar (Bogotá).
$11.4 \%$ víctimas habían sufrido entre 21 a

22 situaciones de acoso; $14.5 \%$ víctimas de 16 a 20 situaciones de acoso; $21 \%$ víctimas de 11 a 15 situaciones de acoso; $21.7 \%$ víctimas de 6-10 situaciones de acoso; 32.4 $\%$ víctimas de 0 a 5 situaciones de acoso.

$\begin{array}{ll} & \text { Nicaragua, } \\ \text { Del Rey y Ortega } & \text { Managua } \\ \text { (2008) } & \mathrm{N}=2.813 \\ & (11-38 \text { años })\end{array}$

Cuevas, Hoyos y Ortiz (2009)

Colombia, Cauca $\mathrm{N}=2.000$ $5^{\circ}$ a $10^{\circ}$ (9-19 años)

Del Río, Bringue, Sádaba y González (2009)
Identificar experiencias de violencia, principalmente la que acontece en el contexto escolar.

Estimar la prevalencia de la intimidación escolar en estudiantes Vallecaucanos.
$35 \%$ implicados en situaciones de acoso;

$12.4 \%$ víctimas; $10.9 \%$ agresores; $11.7 \%$ agresor-victimizado.

$32 \%$ víctimas frecuentes; $90.1 \%$ agresión verbal; $86.2 \%$ agresión social; $71.3 \%$ agresión física; $29.6 \%$ agresión por coacción.
Argentina, Brasil, Chile, Colombia, México Perú y Venezuela $\mathrm{N}=20.941$ (10-18 años)
Explorar el acoso digital a través de internet y teléfonos móviles entre los escolares de Cibervíctimas: Venezuela $17.5 \%$. México $14.7 \%$. Argentina 14.6\%. Chile $13.3 \%$. Perú Argentina, Brasil, Chile, $11.9 \%$. Colombia $11.3 \%$. Brasil $8.4 \%$ Colombia, México, Perú y Venezuela. 
Prevalencia de bullying y cyberbullying en Latinoamérica

una revisión

\begin{tabular}{|c|c|c|c|c|}
\hline Autores (Año) & $\begin{array}{l}\text { Lugar, muestra, } \\
\text { grado y/o (edad) }\end{array}$ & Objetivos de estudio & Resultados bullying & Resultados cyberbullying \\
\hline Flores (2009) & $\begin{array}{l}\text { Bolivia, La Paz } \\
\mathrm{N}=6.700 . \text { Primaria } \\
\text { y Secundaria }\end{array}$ & $\begin{array}{l}\text { Describir la prevalencia del } \\
\text { acoso en Bolivia: víctimas, } \\
\text { agresores y observadores, } \\
\text { tipos de acoso... }\end{array}$ & $\begin{array}{l}50 \% \text { víctimas; } 30 \% \\
\text { agresores; } 60 \% \text { testigos; } \\
59 \% \text { agresión verbal; } 44 \% \\
\text { agresión social; } 40 \% \\
\text { agresión física; } 59 \% \\
\text { agresión psicológica; } 11 \% \\
\text { amenazas o coacciones. Por } \\
\text { áreas geográficas: } \\
\text { Violencia verbal: Chuquisaca } \\
84 \% ; \text { Cochabamba } 70 \% ; \\
\text { Tarija } 68 \% ; \text { Potosí } 67 \% ; \text { Beni } \\
66 \% ; \text { Santa Cruz } 57 \% ; \text { La } \\
\text { Paz y Pando } 52 \% ; \text { y Oruro } \\
26 \% . \\
\text { Violencia social (exclusión, } \\
\text { marginación): } 9 \text { de } 10 \text { en } \\
\text { Oruro; } 7 \text { de } 10 \text { en Potosí; } 5 \text { de } \\
10 \text { en La Paz, Cochabamba, } \\
\text { Santa Cruz, Beni y Pando; y } 4 \\
\text { de } 10 \text { en Tarija y Chuquisaca. } \\
\text { Violencia física: En } \\
\text { Chuquisaca } 6 \text { de } 10 ; \text { de } 5 \text { de } \\
10 \text { en Tarija y Potosí; } 4 \text { de } 10 \\
\text { en Beni, Santa Cruz y La Paz; } \\
\text { y } 3 \text { de } 10 \text { en Cochabamba, }\end{array}$ & $11 \%$ cibervíctimas \\
\hline Lucio López (2009) & $\begin{array}{l}\text { México } \\
\mathrm{N}=1.066 \\
\text { Escuela } \\
\text { preparatoria }\end{array}$ & $\begin{array}{l}\text { Evaluar la prevalencia de } \\
\text { cyberbullying en México }\end{array}$ & & $\begin{array}{l}20 \% \text { ciberagresores: } \\
17.3 \% \text { han insultado; } \\
22.2 \% \text { han amenazado; } \\
10 \% \text { han acosado } \\
\text { sexualmente; } 5.1 \% \text { ha } \\
\text { chantajeado a otro } \\
\text { obligándole a hacer algo } \\
\text { para no difundir cosas } \\
\text { íntimas; } 7.8 \% \text { ha enviado } \\
\text { mensajes desagradables; } \\
11.5 \% \text { ha realizado } \\
\text { comentarios para } \\
\text { difamar; } 10.7 \% \text { ha } \\
\text { difundido rumores para } \\
\text { desprestigiar; } 8.1 \% \text { ha } \\
\text { subido vídeos } \\
\text { desagradables de otro; } \\
7.9 \% \text { ha subido fotos } \\
\text { para desprestigiar o } \\
\text { ridiculizar; } 9.3 \% \text { ha } \\
\text { robado la contraseña; } \\
11.1 \% \text { ha hackeado las } \\
\text { cuentas de otros. }\end{array}$ \\
\hline
\end{tabular}




\begin{tabular}{|c|c|c|c|c|}
\hline Autores (Año) & $\begin{array}{l}\text { Lugar, muestra, } \\
\text { grado y/o (edad) }\end{array}$ & Objetivos de estudio & Resultados bullying & Resultados cyberbullying \\
\hline $\begin{array}{l}\text { Ávila-Toscano, } \\
\text { Osorio-Jaramillo, } \\
\text { Cuello-Vega, } \\
\text { Cogollo-Fuentes y } \\
\text { Causado-Martínez } \\
(2010)\end{array}$ & $\begin{array}{l}\text { Colombia, Córdova } \\
N=120 \\
6^{\circ} \text { a } 9^{\circ} \\
\text { (11- } 16 \text { años) }\end{array}$ & $\begin{array}{l}\text { Identificar la prevalencia de } \\
\text { bullying en adolescentes de } \\
\text { educación básica y media. }\end{array}$ & $\begin{array}{l}69.2 \% \text { implicados: } 30 \% \\
\text { víctimas; } 7.5 \% \text { agresores; } \\
31.7 \% \text { agresores-víctimas } \\
\text { (más varones aunque no hay } \\
\text { diferencias significativas } \\
\text { entre sexos ni en función de } \\
\text { la edad; } 30.8 \% \\
\text { observadores. Mayor } \\
\text { prevalencia verbal y social. }\end{array}$ & \\
\hline
\end{tabular}

Ávila-Toscano, Osorio-Jaramillo, Cuello-Vega, $\mathrm{N}=120$

$6^{\circ}$ a $9^{\circ}$

Causado-Martíne

(2010)
Conocer la prevalencia del

cyberbullying.

$12.9 \%$ cibervíctimas; $5.4 \%$ ciberagresores: móvil $(2.8 \%$ cibervíctimas; $2.1 \%$ ciberagresores); internet ( $4.7 \%$ cibervíctimas; $3.3 \%$ ciberagresores).

\begin{tabular}{|c|c|c|}
\hline $\begin{array}{l}\text { Guaygua y Castillo } \\
(2010)\end{array}$ & Bolivia, La Paz & $\begin{array}{l}\text { Identificar la violencia en las } \\
\text { escuelas Alteñas. }\end{array}$ \\
\hline $\begin{array}{l}\text { Rodríguez-Álvarez } \\
\text { y Delgado de } \\
\text { Briceño (2010) }\end{array}$ & $\begin{array}{l}\text { Venezuela, Caracas } \\
\text { y Miranda } \\
\mathrm{N}=150 \text { profesores } \\
4^{\circ} \text { a } 60\end{array}$ & $\begin{array}{l}\text { Caracterizar las expresiones } \\
\text { de violencia escolar en la } \\
\text { escuela básica Venezolana. }\end{array}$ \\
\hline
\end{tabular}

35\% agresión física.

Las conductas violentas más prevalentes según los profesores son la violencia física como golpes, empujones... (80\%) y violencia verbal como apodos, insultos o amenazas (53\%). La violencia psicológica (humillaciones para minar la autoestima, para crear miedo) es poco identificada por los docentes.

$100 \%$ ha observado alguna conducta de acoso $(77 \%$ son observadores pasivos y $23 \%$ activos en la defensa de la víctima); 8 de cada 10 han sufrido alguna conducta de acoso; la mayor prevalencia es de acoso psicológico, verbal y físico respectivamente.

$50 \%$ han participado en situaciones de acoso escolar; $25.3 \%$ víctimas; $6 \%$ agresores; $18.7 \%$ víctimas agresores; victimización verbal $(21.8 \%$ severa, $23.5 \%$ ocasional); Agresión física (20.2\% severa, $17.3 \%$ ocasional); exclusión social (16.8\% severa, $20.3 \%$ ocasional); agresión psicológica (12.2\% severa, $13.3 \%$ ocasional). 
Prevalencia de bullying y cyberbullying en Latinoamérica

una revisión

\begin{tabular}{|c|c|c|c|c|}
\hline Autores (Año) & $\begin{array}{l}\text { Lugar, muestra, grado y/o } \\
\text { (edad) }\end{array}$ & Objetivos de estudio & Resultados bullying & Resultados cyberbullying \\
\hline $\begin{array}{l}\text { García-Maldonado, Joffre- } \\
\text { Velázquez, Martínez-Salazar y } \\
\text { Llanes-Castillo (2011) }\end{array}$ & $\begin{array}{l}\text { México } \\
\text { Revisión de } 80 \text { trabajos }\end{array}$ & $\begin{array}{l}\text { Describir y precisar sus } \\
\text { diversas características, } \\
\text { señalar algunos aspectos } \\
\text { inherentes al bullying } \\
\text { tradicional y revisar las } \\
\text { diferencias entre ambos } \\
\text { fenómenos. }\end{array}$ & & $\begin{array}{l}40 \% \text { ha tenido algún contacto } \\
\text { con el cyberbullying. } 1 \text { de cada } 4 \\
\text { estudiantes está involucrado en } \\
\text { este problema. El riesgo de ser } \\
\text { cibervictimizado se duplica al } \\
\text { tener un perfil en una red social } \\
\text { electrónica. } 50 \% \text { de las víctimas } \\
\text { no comunica a nadie sobre este } \\
\text { problema. }\end{array}$ \\
\hline
\end{tabular}

México, Guadalajara

Valadez, Amezcua, González, N $=723$ adolescentes Montes y Vargas (2011) (enseñanza media superior)

(12-24 años)
Maltrato Social: exclusión infravalorándoles (5.2\%), impedirles participar (5\%), ignorarlos (5.2\%). Maltrato psicológico: apodos descalificantes $(14.9 \%)$, ridiculización (8.0\%), amenazas (3.7\%), hablar mal (10\%), obligar a hacer cosas $(3.4 \%)$, amenazas con armas (2\%). Maltrato sexual, insultos de Conocer la relación entre el carácter sexual, muecas y gestos maltrato entre iguales y el intento suicida en adolescentes escolarizados

obscenos $(3.1 \%)$, caricias no deseadas (3.4\%), acoso sexual

$(2.8 \%)$. Maltrato a la propiedad: robo de dinero ( $7.3 \%)$; destrozo de cosas (4.9\%). Maltrato físico: los golpes (12.2\%). Un 12.0\% indicó haber sido objeto de más de una forma de maltrato. Asociaron dificultades escolares (desempeño escolar y maltrato) con intento suicida, ideas y pensamientos suicidas, aislamiento social y sentimientos de soledad y abandono.

Determinar la prevalencia de cyberbullying Explorar la asociación con las consecuencias del bullying
$3.5 \%$ cibervíctimas; $2.8 \%$
$24.4 \%$ víctimas; $19.2 \%$ agresores; $32.9 \%$ víctimasagresivas. ciberagresores; $1.3 \%$ cibervíctimas-ciberagresores. Todos los roles se asociaron con problemas para dormir, uso de alcohol y tabaco. 
ópez-Bañuelos et

al. (2012)
México, Tijuana

de 20 a 60

2.160 primaria 661 secundaria 165

preparatoria
Elaborar un diagnóstico de la prevalencia del acoso escolar (bullying) en nivel primaria, secundaria, preparatoria.

Ser víctima: $25.7 \%$ primaria, $18.5 \%$ secundaria, 16.6

preparatoria;

Ser Ofensor: $19.5 \%$ primaria, $28 \%$ secundaria, $31.3 \%$

preparatoria.

Ser testigo: $34.5 \%$ primaria, $48.2 \%$ secundaria, $49.7 \%$

preparatoria;

Ser cómplice: $6.5 \%$ primaria, $13.3 \%$ secundaria, $15.9 \%$

preparatoria.

Víctimas 22.8\%; agresores

$30.5 \%$ admiten que alguna vez

ha agredido de diferentes

formas a un compañero ridiculizándolo, golpeándolo, excluyéndolo, amenazándolo, siendo la más repetitiva la ridiculización con un $44.5 \%$ (agresión social). Las víctimas perciben menor apoyo afectivo. Agresores perciben a su familia moderadamente o gravemente disfuncional.

$\mathrm{N}=304$ estudiantes funcionamiento familiar en (10-18 años) adolescentes percibidos por una institución educativa.

\begin{tabular}{|c|c|c|}
\hline $\begin{array}{l}\text { Avila-Toscano, } \\
\text { Marenco- } \\
\text { Escuderos, y Tilano- } \\
\text { Osorio (2013) }\end{array}$ & $\begin{array}{l}\text { Colombia, } \\
\text { Barranquilla } \\
\mathrm{N}=320 \\
8^{\circ} \text { a } 9^{\circ} \\
\text { (12-16 años) }\end{array}$ & $\begin{array}{l}\text { Identificar prevalencia en } \\
\text { estudiantes de secundaria } \\
\text { implicados en conductas de } \\
\text { acoso. }\end{array}$ \\
\hline $\begin{array}{l}\text { Silva-Villarreal, } \\
\text { Castillo, Eskildsen, } \\
\text { Vidal, Mitre, y } \\
\text { Quintero (2013) }\end{array}$ & $\begin{array}{l}\text { Panamá } \\
\mathrm{N}=472 \\
\text { Estudiantes } \\
\text { universitarios } \\
1^{\circ} \text { a } 5 \text { to }\end{array}$ & $\begin{array}{l}\text { Determinar la prevalencia de } \\
\text { bullying entre estudiantes } \\
\text { panameños de la carrera de } \\
\text { Medicina. }\end{array}$ \\
\hline
\end{tabular}

Vega-López, González-Pérez y Quintero-Vega (2013)

\section{$18.8 \%$ víctimas; $21.6 \%$} agresores; $36.3 \%$ agresorvíctima; $23.4 \%$ observador.

$39.8 \%$ implicados en situaciones de bullying; $15 \%$ de víctimas; $6.6 \%$ de agresores; $18.2 \%$ de víctimas-agresivas.
$14.3 \%$ cibervíctimas.

Principal forma de agresión: transmisión de textos e imágenes insultantes por el móvil. $51 \%$ mensajes de texto o imágenes negativas; $37 \%$ mensajería instantánea; $30 \%$ a través de correo electrónico. Mayor prevalencia en chicos. 
Prevalencia de bullying y cyberbullying en Latinoamérica

\begin{tabular}{|c|c|c|c|c|}
\hline Autores (Año) & $\begin{array}{l}\text { Lugar, muestra, } \\
\text { grado y/o (edad) }\end{array}$ & Objetivos de estudio & Resultados bullying & Resultados cyberbullying \\
\hline $\begin{array}{l}\text { Vega-López, } \\
\text { González-Pérez, } \\
\text { Valle-Barbosa, } \\
\text { Flores-Villavicencio } \\
\text { y Vega-López } \\
\text { (2013) }\end{array}$ & $\begin{array}{l}\text { México, } \\
\text { Guadalajara } \\
\mathrm{N}=1.706 \\
\text { (11-16 años) }\end{array}$ & $\begin{array}{l}\text { Determinar la prevalencia } \\
\text { de víctimas de acoso escolar } \\
\text { en alumnos de escuelas } \\
\text { secundarias. }\end{array}$ & $17.6 \%$ víctimas & \\
\hline $\begin{array}{l}\text { Cassiani-Miranda, } \\
\text { Gómez-Alhach, } \\
\text { Cubides-Munévar y } \\
\text { Hernández-Carrillo } \\
\text { (2014) }\end{array}$ & $\begin{array}{l}\text { Colombia, Cali } \\
N=198 \\
6^{\circ} \text { a } 9^{\circ} \\
(11-16 \text { años })\end{array}$ & $\begin{array}{l}\text { Determinar prevalencia de } \\
\text { bullying y factores } \\
\text { relacionados. }\end{array}$ & $\begin{array}{l}20.3 \text { \% víctimas. Ser víctima se } \\
\text { asoció con disfunción familiar } \\
\text { leve y severa y síntomas } \\
\text { clínicos de ansiedad. }\end{array}$ & \\
\hline
\end{tabular}

Bolivia, Santa

Egüez y Cruz

Schulmeyer (2014) $\quad \mathrm{N}=1.610$

(12-16 años)
Colombia,
González, Mariaca y Medellín Arias (2014)

3.373 estudiantes $6^{\circ}$ a $11^{\circ}$
$10 \%$ víctimas: Violencia verbal: $40 \%$ afirmó que hablaban mal de ellos, $37 \%$ recibía insultos $\mathrm{y} / \mathrm{o}$ apodos ofensivos. Violencia de exclusión: $29 \%$ dijo que no se les dejaba participar, $22 \%$ era ignorado $\mathrm{y} / \mathrm{o}$ marginado. Coacciones: $11 \%$ recibió amenazas, $5 \%$ fue obligado a hacer cosas que no quería hacer y un $3 \%$ fue atacado con algún arma.

Identificar la prevalencia de $4 \%$ agresores: $33 \%$ insulta, bullying y cyberbullying

$27 \%$ habla mal de alguien, $25 \%$ pone apodos ofensivos, $22 \%$ ignora, margina a sus víctimas, $19 \%$ no deja participar, $13 \%$ esconde cosas, $7 \%$ rompe cosas y $5 \%$ roba cosas, $9 \%$ amenaza, $3 \%$ obliga sus compañeros a hacer cosas que no quieren, $2 \%$ amenaza con alguna arma, $3 \%$ acosa sexualmente. El $12 \%$ admite agredir físicamente a sus compañeros.

$\begin{array}{lll}\text { Forlim, Stelko- } & \text { Brasil } & \text { Describir la prevalencia y las } \\ \text { Pereira, y Williams } & \mathrm{N}=348 & \text { relaciones entre los tipos de } \\ 6^{\circ} \text { a } 9^{\circ} & \text { implicación en casos de } \\ (2014) & (13-18 \text { años }) & \text { bullying. }\end{array}$

$16 \%$ víctimas, $11 \%$ agresores y $23 \%$ víctimas-agresivas.

Presencia de bullying en el $37.6 \%$ de los estudiantes, expresado en comportamientos de Identificar la prevalencia del intimidación o agresión verbal, bullying en Medellín. física y psicológica en ambos sexos $y$ todos los grados escolares. Las formas de agresión más frecuentes son la verbal y la psicológica.
$16 \%$ cibervíctimas hostigados a través del móvil o internet (facebook, e-mail, etc.); $12 \%$ ciberagresores.
Lanzillotti y

Korman (2014)
Argentina, Buenos

Aires

$\mathrm{N}=36$

(12-18 años)
Describir y caracterizar el cyberbullying
23.5\% había sido hostigado a través del móvil y $44.1 \%$ a través de internet. 


\begin{tabular}{|c|c|c|}
\hline Autores (Año) & $\begin{array}{l}\text { Lugar, muestra, } \\
\text { grado y/o (edad) }\end{array}$ & Objetivos de estudio \\
\hline $\begin{array}{l}\text { Ponce y Cucunubà } \\
\text { (2014) }\end{array}$ & $\begin{array}{l}\text { Colombia } \\
\mathrm{N}=2.259 \text { de } \\
7^{\circ} \text { a } 9^{\circ}\end{array}$ & $\begin{array}{l}\text { Identificar el bullying escolar } \\
\text { en centros educativos } \\
\text { distritales }\end{array}$ \\
\hline $\begin{array}{l}\text { Prodócimo, Cerezo } \\
\text { y Arence } \\
\text { Prodócimo, Cerezo } \\
\text { y Arence }(2014)\end{array}$ & $\begin{array}{l}\text { Brasil } \\
\mathrm{N}=2.793 \\
(10-18 \text { años) }\end{array}$ & $\begin{array}{l}\text { Conocer la incidencia del } \\
\text { acoso escolar en las escuelas } \\
\text { brasileñas. }\end{array}$ \\
\hline
\end{tabular}

$\begin{array}{lll}\text { Santoyo y Frías } & \begin{array}{l}\text { México } \\ \text { Encuesta }\end{array} & \begin{array}{l}\text { Examinar la prevalencia de } \\ \text { acoso escolar (bullying) } \\ \text { entre estudiantes de nivel } \\ \text { (2014) }\end{array} \\ & \text { macional } & \end{array}$

Silva, Andrade, Brasil Leichsenring y Hirle $\mathrm{N}=439$ (2014) $5^{\circ}$ grado
Caracterizar el bullying e intervenir para la mejora
Resultados bullying

$30 \%$ de víctimas, en el que participan alumnos y profesores de manera activa o pasiva.

\section{$31.4 \%$ implicados; $13.6 \%$}

víctimas (no diferencias entre sexos); $8.9 \%$ agresores (más varones); $7.8 \%$ víctimasagresivas (no diferencias de género).

$25.8 \%$ víctimas; $18.4 \%$ agresores ( $15.4 \%$ víctimas puras; $10.4 \%$ víctimasagresoras; $8 \%$ agresores puros). Víctimas: $8.7 \%$ acoso físico; $19.2 \%$ acoso emocional; $5.4 \%$ acoso sexual. Agresores: $12.2 \%$ acoso físico; $9 \%$ acoso emocional; $3.2 \%$ acoso sexual.

49.9\% víctimas; $34.9 \%$ agresores.

Tipos: insultos (11.8\%), golpes (8.0\%), bofetadas $(7.3 \%)$, patadas (1.4\%), empujones (3.4\%), palabras ofensivas (18.0\%) repetidas veces. Los sentimientos más predominantes en las víctimas son los de tristeza (19.8\%) y de remordimiento en los agresores (47.4\%).

$\begin{array}{ll}\text { Varela, Pérez, } & \text { Chile } \\ \text { Schwaderer, } & \mathrm{N}=1.357 \\ \text { Astudillo y } & 7^{\circ} \text { básico a } 4^{\circ} \\ \text { Lecannelier (2014) } & \begin{array}{l}\text { secundaria } \\ \text { (11-16 años) }\end{array}\end{array}$

Describir la prevalencia de cyberbullying.
$11.4 \%$ cibervíctimas; $12.5 \%$ ciberagresores.
$4.6 \%$ víctimas de estudiantes Portorriqueños que viven en Puerto Rico; $15.2 \%$ víctimas de estudiantes Portorriqueños que viven en el Sur del Bronx (Nueva York). Factores de Riesgo: pobre ajuste social, bajo rendimiento académico, disciplina parental dura, ambiente escolar, exposición a la violencia y contexto de culturización.

$7 \%$ víctima, $12 \%$ agresores, 4\% agresor-víctimas; menor autoestima víctima, mayor depresión agresor-víctima, mayor ansiedad agresores.
$\mathrm{N}=2.491$

(10-13 años)
Examinar factores

contuales familiar asociados con el acoso a otros niños en dos sitios diferentes.

Explorar el porcentaje de

Resett (2015)

$\mathrm{N}=502$

$1^{\circ}$ a $5^{\circ}$ secundaria

agresivas, y no involucrados.
México, Cuenca

Robalino (2015) $\quad \mathrm{N}=493$
Describir la prevalencia de bullying en centros rurales del cantón cuenca.
18.5 víctimas ( $20 \%$ mujeres, $17.3 \%$ varones). 
Prevalencia de bullying y cyberbullying en Latinoamérica

\begin{tabular}{|c|c|c|c|c|}
\hline Autores (Año) & $\begin{array}{l}\text { Lugar, muestra, } \\
\text { grado y/o (edad) }\end{array}$ & Objetivos de estudio & Resultados bullying & Resultados cyberbullying \\
\hline $\begin{array}{l}\text { Shephard, Ordónez } \\
\text { y Mora (2015) }\end{array}$ & $\begin{array}{l}\text { Ecuador, Cuenca } \\
\mathrm{N}=885 \\
2^{\circ} \text { a } 9^{\circ} \text { primaria. }\end{array}$ & $\begin{array}{l}\text { Establecer la prevalencia del } \\
\text { acoso escolar. }\end{array}$ & $\begin{array}{l}10.6 \% \text { víctimas en un centro y } \\
9.1 \% \text { víctimas en otro centro. } \\
\text { Víctimas potenciales } 34.7 \text { y } \\
38.4 \% \text { respectivamente. Robo } \\
6.7 \% \text { y } 8.4 \% \text {; Daño físico } 10.9 \text { y } \\
13.9 \text {; Apodos y burlas } 39.4 \% \text { y } \\
28.5 \text {. }\end{array}$ & \\
\hline
\end{tabular}

$\begin{array}{lll} & \text { Chile, Arequipa } & \\ \mathrm{N}=3.979 & \text { Determinar la prevalencia del } \\ \text { Bellido et al. (2016) } & 1^{\circ} \text { a } 5^{\circ} \text { de } & \text { bullying en el área } \\ & \text { secundaria } & \text { metropolitana de la ciudad de } \\ & (11-19 \text { años }) & \text { Arequipa. }\end{array}$

Colombia,

Beltrán, Torrado y

Vargas (2016)

Bucaramanga

$\mathrm{N}=1.776$

$4^{\circ}$ a $11^{\circ}$

(8-18 años)

$\begin{array}{lll} & \begin{array}{l}\text { Colombia, } \\ \text { Bucaramanga }\end{array} & \begin{array}{l}\text { Determinar la prevalencia del } \\ \text { hostigamiento escolar }\end{array} \\ \text { Beltrán, Torrado y } & \mathrm{N}=1.776 & \begin{array}{l}\text { presente en las instituciones } \\ \text { Vargas (2016) }\end{array} \\ & 4^{\circ} \text { a } 11^{\circ} & \text { educativas públicas de } \\ & (8-18 \text { años }) & \text { Bucaramanga-Colombia. }\end{array}$

$14.2 \%$ agresores (matones e

inteligentes: más varones), $25 \%$ víctimas (pasivas: más mujeres, y provocadoras: más varones), $11.5 \%$ agresor-víctima. $49.4 \%$ observadores (más mujeres).

$8.1 \%$ víctimas. $5 \%$ cibervíctimas.

Colombia, Popayán

$\mathrm{N}=1.300$

$\mathrm{N}=$ Padres 513

$\mathrm{N}=$ Docentes 81

$6^{\circ}$ a $8^{\circ}$

(9-17 años)
Describir el fenómeno de la intimidación escolar.

\section{4\% intimidación física}

(golpes, patadas, sustracción de objetos); $70 \%$ intimidación psicológica (gritar, apodos, coaccionar, amenazar y excluir); $35.1 \%$ los profesores consideran el problema grave; $31.4 \%$ de los padres consideran muy grave el problema.

Total muestra: $37.8 \%$ víctimas.

47.8\% Perú. 31.6\% Bolivia.

$31.6 \%$ Honduras. $19.5 \%$ Costa

Rica. 19.3\% Uruguay.

8.2\% agresores con predominio en la conducta agresora masculina.

$41.9 \%$ bullying; $23.4 \%$ víctimas, $18.7 \%$ cyberbullying; $4.5 \%$ agresores, $14 \%$ agresores- $10.7 \%$ cibervíctima, victimizados. Existe coocurrencia entre bullying y cyberbullying. $2.5 \%$ ciberagresor, $5.5 \%$ Identificar la prevalencia de bullying y cyberbullying.
Identificar la prevalencia de escuelas secundarias públicas de la Guadalaiara. ciberagresorvictimizado.
Romera y Ortega-

Ruiz (2017)

\section{México,}

$\mathrm{N}=1.706$

adolescentes

(12-15 años)

Colombia

(11-19 años)
$13 \%$ víctimas, $31 \%$ agresores.

Mayor ansiedad: víctimasagresivas y víctimas.

\section{México}

$\mathrm{N}=1.190$

Barrera (2017)
574 primaria y 616 secundaria (8-16 años)

\section{Conocer las diferencias entre} grupos de alumnos en edad bullying, en función de la ansiedad. 
Redondo, Luzardo- Colombia, Briceño, GarcíaLizarazo e Inglés (2017) Bucaramanga $\mathrm{N}=637$ 17 años
Determinar la prevalencia del ciberbullying y conocer el impacto psicológico.

$27.5 \%$ cibervíctimas (una o más veces); $26.7 \%$ ciberagresores (una o más veces) en el último año. Impacto psicológico en cibervíctimas y ciberagresores (contigencias con síntomas psicopatológicos del SCL90)

$8 \%$ ciberagresores; $4 \%$ fueron agresores y ciberagresores.

Comparar bullying y

Argentina cyberbullying en los problemas $\mathrm{N}=898 \quad$ emocionales, $\mathrm{y}$ la personalidad de aquellos que participan en la

perpetración de acoso

tradicional. $\begin{array}{ll}\text { Guadix (2017) } & 7^{\circ} \text { a } 12^{\circ} \\ & (12-19 \text { años })\end{array}$

$\begin{array}{ll}\text { Guadix (2017) } & 7^{\circ} \text { a } 12^{\circ} \\ & (12-19 \text { años })\end{array}$

$6 \%$ agresores.
Analizar la incidencia de los estilos de crianza en la presencia de cyberbullying en estudiantes de secundaria.
Ciberagresores mostraron menos depresión y ansiedad, menor neuroticismo y más amabilidad que los agresores.

$26 \%$ cibervíctimas, $15.7 \%$ ciberagresores, $55 \%$ observador. Relación entre ser cibervíctima y estilo de crianza autoritario, y entre ser ciberagresor y estilos democrático y negligente.
Gordillo-Rondón, Redondo y Luzardo (2017) (11-17años) $\mathrm{N}=281$ $6^{\circ}$ a $11^{\circ}$

Colombia, Bucaramanga
$29.5 \%$ víctimas. $8.4 \%$ agresores. La mayor prevalencia en acoso psicológico (23.3\%). La mayoría de los implicados son chicos.
Aportar la prevalencia de victimización y agresión de bullying.
De Castro, Leite, Brasil
Nascimento, Nunes, $\mathrm{N}=678$ $y$ de Sales (2018) $\quad 6^{\circ}$ a $9^{\circ}$
Mallmann, Lisboa, y $\begin{aligned} & \text { Brasil } \\ & \mathrm{N}=273\end{aligned}$

Calza (2018)

(13-18 años)
Evaluar la prevalencia de cyberbullying en adolescentes de Rio Grande do Sul
$58 \%$ cibervíctimas (una o más veces). Estrategias de afrontamiento de autocontrol, soporte social y escape-huida mayores en víctimas versus no involucrados. La estrategia de confrontación fue más utilizada por las víctimasagresores.

\section{Argentina, Bahía}

Blanca

Zalba et al. (2018)

$22.1 \%$ agresores frecuentes y

Examinar la asociación entre bullying y rendimiento académico
$30.12 \%$ ocasional. No encontraron relación entre bullying y rendimiento académico. 
Las prevalencias encontradas (ver Tabla 1) son relevantes. Una síntesis de los resultados de los estudios realizados en este periodo (2005-2018) se presenta a continuación por países (ordenados en función de la cantidad de estudios realizados en cada país) y, después, se exponen las conclusiones globales para el conjunto de América Latina.

Colombia es el país Latinoamericano en el que más estudios de prevalencia se han realizado, en concreto se han encontrado 14 investigaciones, $\mathbf{9}$ de bullying, $\mathbf{3}$ de cyberbullying, y $\mathbf{2}$ que aportan datos de ambas modalidades de acoso. En bullying se ha identificado entre 8.1\% (Beltrán, Torrado, \& Vargas, 2016) y 37.6\% (González, Mariaca, \& Arias., 2014) de víctimas, entre 4.5\% (Herrera-López, Romera, \& Ortega-Ruiz, 2017) y $\mathbf{3 0 . 5 \%}$ (Uribe, Orcasita, \& Aguillón, 2012) de agresores, y entre 14\% (Herrera-López, Romera, \& Ortega-Ruiz, 2017) y 36.3\% (Ávila-Toscano, Marenco-Escuderos, \& Tilano-Osorio., 2013) de víctimas-agresivas. En cyberbullying, los porcentajes de cibervíctimas oscilan entre $\mathbf{5 \%}$ (Beltrán, Torrado, \& Vargas, 2016) y $\mathbf{2 7 . 5 \%}$ (Redondo, Luzardo-Briceño, García-Lizarazo, \& Inglés, 2017), y de ciberagresores entre $\mathbf{2 . 5 \%}$ (Herrera-López, Romera, \& Ortega-Ruiz, 2017) y $\mathbf{2 6 . 7 \%}$ (Redondo, Luzardo-Briceño, García-Lizarazo, \& Inglés, 2017); además un estudio (Herrera-López, Romera, \& Ortega-Ruiz, 2017) identifica un $\mathbf{5 . 5 \%}$ de cibervíctimas-ciberagresoras.

México es otro de los países de Latinoamérica donde más se ha estudiado la prevalencia del bullying y el cyberbullying. Se han llevado a cabo 12 estudios, 7 de bullying, $\mathbf{4}$ de cyberbullying, y 1 que aporta datos de bullying y cyberbullying. En bullying se evidencian porcentajes de victimización que oscilan entre 13\% (Mendoza, Rojas, \& Barrera, 2017) y $\mathbf{2 5 . 8 \%}$ (Santoyo \& Frías, 2014) de víctimas. En perpetración de bullying se encuentran porcentajes entre $\mathbf{8 . 2 \%}$ (VegaLópez \& González-Pérez, 2016) y 31.3\% (Mendoza, Rojas, \& Barrera, 2017) de agresores. Vega-López \& González-Pérez (2016) muestran mayores niveles de perpetración en chicos, porcentajes entre $\mathbf{3 4 . 5 \%}$ y $\mathbf{4 9 . 7 \%}$ que han sido testigos de agresiones en la escuela, además, el porcentaje de víctimas disminuye con la edad (desde primaria hasta preparatoria), pero aumenta el de agresores. En el rol de víctimasagresivas los porcentajes oscilan entre $\mathbf{1 0 . 8 \%}$ (Santoyo \& Frías, 2014) y $\mathbf{3 2 . 9 \%}$ (García-Maldonado, y otros, 2012). En cyberbullying, el porcentaje de cibervíctimas varía entre $\mathbf{3 . 5 \%}$ (García-Maldonado, y otros, 2012) y $\mathbf{4 0 \%}$ (García-Maldonado, Joffre-Velázquez, MartínezSalazar, \& Llanes-Castillo, 2011). El porcentaje de ciberagresores fluctúa entre $\mathbf{2 . 8 \%}$ (García-Maldonado, y otros, 2012) y $\mathbf{2 0 \%}$ (Lucio-López, 2009), encontrando un $\mathbf{1 . 3} \%$ de cibervíctimas-ciberagresores (Lucio López, 2009).

En Argentina se han llevado a cabo $\mathbf{6}$ estudios, $\mathbf{3}$ de bullying, $\mathbf{2}$ de cyberbullying, y $\mathbf{1}$ que aporta información de bullying y cyberbullying. En bullying se identifica un porcentaje de víctimas que oscila entre $\mathbf{7 \%}$ (Resett, 2015) y $\mathbf{2 1 . 1 \%}$ (Zalba, y otros, 2018), un porcentaje de agresores entre 6\% (Resett \& Gámez-Guadix, 2017) y $\mathbf{1 6 . 6 \%}$ (Kornblit \& Adaszko, 2007), así como 4\% de víctimas-agresivas (Resett, 2015). En cyberbullying se encuentra entre un $\mathbf{1 4 . 6 \%}$ (del Río et al., 2009) de cibervíctimas, a un $\mathbf{2 3 . 5 \%}$ cibervíctimas de móvil y $\mathbf{4 4 . 1 \%}$ de Internet (Lanzillotti \& Korman, 2014). Con respecto a los ciberagresores, el $\mathbf{8 \%}$ de los participantes se han identificado como ciberacosadores (Resett \& Gámez-Guadix, 2017).

En Brasil se han realizado $\mathbf{5}$ estudios de bullying y $\mathbf{2}$ de cyberbullying. El estudio de Abramovay (2005) constató que un $\mathbf{8 3 . 4 \%}$ de los participantes percibe violencia en la escuela. En bullying, las prevalencias de victimización de estos estudios oscilan entre $\mathbf{1 6 \%}$ (Forlim, Stelko-Pereira, \& Williams, 2014) y 49.9\% (Silva, Andrade, Leichsenring, \& Hirle, 2014) de víctimas. En perpetración de bullying, los porcentajes de agresores varían entre $\mathbf{8 . 4 \%}$ (Prodócimo, Cerezo, \& Arense, 2014) y $\mathbf{3 4 . 9 \%}$ (Silva, Andrade, Leichsenring, \& Hirle, 2014). Además, los porcentajes de víctimas-agresivas varían entre $\mathbf{7 . 8 \%}$ (Prodócimo, Cerezo, \& Arense, 2014)y 23\% (Forlim, Stelko-Pereira, \& Williams, 2014). En general los chicos son más agresores (De Castro, Leite, Nascimiento, Nunes, \& De sales., 2018). En cyberbullying, la cibervictimización oscila entre un $\mathbf{8 . 4 \%}$ (Del Río, Bringue, Sádaba, \& González, 2009) a un 58\% (Mallmann, Lisboa, \& Calza, 2018) de cibervíctimas.

Bolivia cuenta con $\mathbf{4}$ estudios, 2 de bullying y $\mathbf{2}$ sobre cyberbullying. En bullying victimización han encontrado entre un 10\% (Egüez \& Schulmeyer, 2014) y un $\mathbf{5 0 \%}$ (Flores, 2009) de víctimas, así como entre $\mathbf{4 \%}$ (Egüez \& Schulmeyer, 2014) y $\mathbf{3 0 \%}$ (Flores, 2009) de agresores. En cyberbullying, se encuentran porcentajes de cibervíctimas entre $\mathbf{1 1} \%$ (Flores, 2009) y $\mathbf{1 6 \%}$ (Egüez \& Schulmeyer, 2014), así como $\mathbf{1 2} \%$ de ciberagresores.

En Perú se identifican también $\mathbf{4}$ estudios, $\mathbf{2}$ sobre bullying y $\mathbf{2}$ de cyberbullying. Ávila, Becerra, Vásquez, \& Becerra (2011) encontraron que el $\mathbf{1 0 0 \%}$ de los estudiantes de $\mathbf{1 2}$ a $\mathbf{1 6}$ años decían haber sido testigo de situaciones de bullying alguna vez, y el $\mathbf{8 0 \%}$ informaron haber sufrido alguna conducta de acoso cara-a-cara. En cibervictimización los resultados oscilan entre $\mathbf{1 1 . 9 \%}$ (Del Río, Bringue, Sádaba, \& González, 2009) y $\mathbf{1 2 . 9 \%}$ (García, y otros, 2010) cibervíctimas, y $\mathbf{5 . 4 \%}$ ciberagresores (García, y otros, 2010).

En Chile se han realizado $\mathbf{3}$ estudios, 1 sobre bullying y $\mathbf{2}$ de cyberbullying. Bellido y otros (2016) han encontrado un $\mathbf{2 5 \%}$ de víctimas, $\mathbf{1 4 . 2 \%}$ de agresores y $\mathbf{1 1 . 5} \%$ víctimas-agresivas, hallando mayor porcentaje de agresores varones. En cibervictimización se ha encontrado un porcentaje de cibervíctimas que oscila entre $\mathbf{1 1 . 4 \%}$ (Varela, Pérez, Schwaderer, Astudillo, \& Lecannelier, 2014) y 13.3\% (Del Río, Bringue, Sádaba, \& González, 2009).

En Nicaragua se han llevado a cabo $\mathbf{3}$ estudios sobre bullying. La victimización en Nicaragua fluctúa entre un 12.4\% (Del Rey \& Ortega, 2008), y un $\mathbf{2 5 . 3 \%}$ (Romera, Del Rey, \& Ortega, 2011) de víctimas, mientras que los agresores oscilan entre 6\% (Romera, Del Rey, \& Ortega, 2011), y $\mathbf{1 0 . 9 \%}$ (Del Rey \& Ortega, 2008), y las víctimas-agresivas entre $\mathbf{1 1 . 7 \%}$ (Del Rey \& Ortega, 2008), y $\mathbf{1 8 . 7 \%}$ (Romera, Del Rey, \& Ortega, 2011). No se han identificado estudios que aporten datos sobre cyberbullying.

En Venezuela se han detectado $\mathbf{2}$ estudios, $\mathbf{1}$ de bullying y $\mathbf{1}$ de cyberbullying. Rodríguez-Álvarez y Delgado de Briceño (2010) encontraron que el $\mathbf{8 0} \%$ habían sufrido violencia física y $\mathbf{5 3 \%}$ violencia verbal. Además Del Río, Bringue, Sádaba, \& González (2009), encontraron $\mathbf{1 7 . 5} \%$ de cibervíctimas.

En otros países como Panamá, Ecuadory Puerto Rico, únicamente se encuentra un estudio respectivamente sobre bullying. En Panamá se identifican $\mathbf{1 5 \%}$ de víctimas, $6 \%$ de agresores y $\mathbf{1 8 . 2} \%$ de víctimasagresivas (Silva-Villarreal, y otros, 2013). En Ecuador el estudio de Shephard, Ordóñez, \& Mora (2015) encuentra entre $\mathbf{9 . 1 \%}$ y $\mathbf{1 0 . 6 \%}$ de víctimas. Y en Puerto Rico hallan $\mathbf{4 . 6 \%}$ de víctimas portorriqueñas que viven en Puerto Rico y $\mathbf{1 5 . 2 \%}$ de víctimas portorriqueños que viven en el Sur del Bronx (Nueva York), lo que pone de relieve la influencia del contexto socio-cultural. 


\section{Discusión}

Los resultados de las investigaciones no son fácilmente comparables por varias razones: (1) Algunos estudios aportan porcentajes de víctimas y agresores severos (que sufren y/o realizan las conductas muy frecuentemente), mientras que otros informan del porcentaje de estudiantes que sufren/realizan esas conductas una o más veces (integrando las conductas ocasionales y frecuentes); (2) Las investigaciones utilizan distintos instrumentos de evaluación del bullying y cyberbullying que no siempre miden las mismas conductas, especialmente en cyberbullying; (3) Las muestras de los estudios varían mucho en cuanto a la edad (niños, adolescentes y jóvenes), y la edad es otro factor de influencia; y (4) El intervalo de tiempo considerado en los distintos estudios difiere (algunos preguntan en qué medida se ha sufrido/realizado ese tipo de conductas desde que comenzó el curso, en el último año, en los últimos dos o tres meses, otros no establecen ninguna limitación temporal...). Por todo ello, solo se puede aportar una horquilla de porcentajes de victimización y perpetración. No obstante, los resultados ponen de relieve que el problema es digno de consideración, lo que permite enfatizar la necesidad de evaluación, prevención e intervención.

Los resultados de la prevalencia del bullying/cyberbullying en Latinoamérica tienen implicaciones prácticas y permiten sugerir la necesidad de implementar medidas de tipo educativo así como de ampliarla concienciación social sobre estefenómeno para quetodos los sectores tomen un papel activo en el tema del bullying/cyberbullying. Estas medidas deberían implicar a las familias, escuelas, estudiantes y a otras entidades sociales. Estas propuestas de intervención deben realizarse en tres niveles: prevención, intervención primaria (cuando se trata de situaciones de acoso puntuales) e intervención secundaria (cuando se trata de situaciones consolidadas).

En todos los centros escolares debe haber un protocolo de actuación para los casos de acoso escolar, así como un plan de prevención de la violencia y promoción de la convivencia escolar. Todos los estudiantes deben participar en programas de intervención preventiva con el objeto de que la prevalencia del bullying en todas sus modalidades sea la menor posible. En líneas generales, los programas de intervención psicológica en contextos educativos, que tienen como finalidad prevenir y reducir el bullying y el cyberbullying, deben promover una mejora del clima social del aula y potenciar el desarrollo de la conducta prosocial, las habilidades sociales, la comunicación, la resolución pacífica de los conflictos, la capacidad de empatía, el control de la ira, el respeto de las diferencias... (Garaigordobil \& Martínez-Valderrey, 2018). Esta perspectiva preventiva ha sido también enfatizada por otros investigadores. Por ejemplo, (Gómez, 2014) considera que la Psicología debe contemplar estrategias efectivas de promoción de conductas sociales y de prevención de conductas violentas, fomentando el incremento de la capacidad de definición y comprensión de un problema, las consecuencias de la conducta violenta y el conocimiento de estrategias pacíficas y adecuadas para su resolución.

\section{Conclusiones}

La mayor parte de los estudios Latinoamericanos aportan información sobre bullying (porcentaje de víctimasy agresores), aunque en los últimos años se están realizando cada vez más investigaciones sobre el cyberbullying. Colombia y México son los países que cuentan con mayor número de estudios de bullying y cyberbullying (p.e. (ÁvilaToscano, Marenco-Escuderos, \& Tilano-Osorio., 2013; Beltrán, Torrado, \& Vargas, 2016; Cuevas, Hoyos, \& Ortiz, 2009; Del Río, Bringue, Sádaba, \& González, 2009; López-Bañuelos, y otros, 2012; Robalino, 2015; Uribe, Orcasita, \& Aguillón, 2012). En todos los países de Latinoamérica se constata una alta prevalencia tanto de bullying ocasional/frecuente (víctimas entre $\mathbf{4 . 6 \%}$ en Puerto Rico y $\mathbf{5 0 \%}$ en Bolivia; agresores entre $\mathbf{4} \%$ en Bolivia y $\mathbf{3 4 . 9 \%}$ en Brasil) como de cyberbullying ocasional/ frecuente (cibervíctimas entre $\mathbf{3 . 5 \%}$ en México y $\mathbf{1 7 . 5 \%}$ en Venezuela; ciberagresores entre $\mathbf{2 . 5} \%$ en Colombia y $\mathbf{5 8} \%$ en Brasil). Todos los estudios encontrados indican que existe una significativa prevalencia de acoso entre iguales en todos los contextos geográficos, culturales y educativos de Latinoamérica. Además, el tipo de bullying más frecuente es el verbal, seguido por el psicológico y el físico (p.e. (Egüez \& Schulmeyer, 2014; Erazo, 2016; Silva, Andrade, Leichsenring, \& Hirle, 2014). Entre las conductas de cyberbullying más frecuentes identificadas en esta revisión caben destacar: envío de mensajes desagradables, insultos, amenazas, realizar comentarios y hacer circular rumores para desprestigiar o ridiculizar a la víctima, difundir fotos y videos ofensivos para la víctima, robo de la contraseña... (p.e. (Egüez \& Schulmeyer, 2014; Lanzillotti \& Korman, 2014; Vega-López., González-Pérez, ValleBarbosa, Flores-Villavicencio, \& Vega-López, 2013). En general se encuentran más agresores varones (p.e. (González, Mariaca, \& Arias., 2014; Prodócimo, Cerezo, \& Arense, 2014; Robalino, 2015). Aunque el bullying cara-a-cara, especialmente la violencia física, disminuye con la edad, el cyberbullying, que emerge más tardíamente, sigue presente en la adolescencia tardía y la juventud (p.e. (López-Bañuelos, y otros, 2012).

Los resultados confirman que la prevalencia de bullying y, también aunque en menor medida de cyberbullying, en Latinoamérica es relevante, siendo similar a la identificada en otras revisiones de estudios que incluyen investigaciones de prevalencia de todos los países del mundo (ver revisión (Garaigordobil, 2017b). No obstante, se evidencian discrepancias en los porcentajes de víctimas y agresores que identifican los estudios, éstos varían, no son homogéneos, por lo que resulta difícil aportar una cifra concreta que refleje la prevalencia exacta en niños, adolescentes y jóvenes.

\section{Aportaciones, limitaciones, y líneas futuras de investigación}

El trabajo aporta información relevante sobre la presencia del bullying y cyberbullying en Latinoamérica. Cabe destacar como limitación del estudio la ausencia de procesamiento de estos datos mediante un metaanálisis. Confirmada la alta existencia de actitudes violentas cara-a-cara o con medios electrónicos (internet, teléfonos móviles...) entre los estudiantes latinoamericanos, se sugiere la necesidad de seguir investigando este fenómeno, por ejemplo, explorando factores de protección y de riesgo, identificando pautas positivas de educación parental y de actuación de los docentes, diseñando y evaluando programas de prevención a lo largo de toda la escolaridad, evaluando los protocolos que se aplican cuando aparece un caso de bullying/cyberbullying. 


\section{Referencias}

Abramovay, M. (2005). Victimización en las escuelas. Ambiente escolar, robos y agresiones físicas. Revista Mexicana de Investigación Educativa, 10(26), 833-864. Obtenido de http://www.redalyc.org/articulo.oa?id=14002611

Ávila, M., Becerra, S., Vásquez, J., \& Becerra, S. (2011). Acoso escolar en instituciones educativas de la ciudad de Huancayo en el 2011. Revista Apuntes de Ciencia \& Sociedad, 1(2), 83-91. Obtenido de http://journals. continental.edu.pe/index.php/apuntes/article/view/28

Ávila-Toscano, J. H., Marenco-Escuderos, A. D., y Tilano-Osorio M.A. (2013). Redes de iguales y acoso escolar: evaluación desde el análisis de redes sociales. Psychologia. Avances de la Disciplina, 7(1), 53-64. http://www.scielo.org.co/ pdf/psych/v7n1/v7n1a06.pdf

Ávila-Toscano, J. H., Osorio Jaramillo, L., Cuello Vega, K., Cogollo-Fuentes, N., \& Causado-Martínez, K. (2010). Conducta bullying y su relación con la edad, género y nivel de formación en adolescentes. Revista Psicogente, 13(23), 13-26. Obtenido de http://www.redalyc.org/pdf/4975/497552352002.pdf

Bellido, F., Rivera, R., Salas, J., Bellido, V., Peña, N., Villasante, G., \& Casapía, Y. (2016). Influencia de los pares en la manifestación del bullying en estudiantes de secundaria en Arequipa Metropolitana. Revista Interacciones , 2(1), 33-42. doi:10.24016/2016.v2n1.20

Beltrán V., Y. I., Torrado D., O. E., \& Vargas B., C. G. (2016). Prevalencia del Hostigamiento Escolar en las Instituciones Públicas de BucaramangaColombia. Revista Sophia, 12(2), 173-86. doi:https://doi.org/10.18634/ sophiaj.12v.2i.233

Blanco-Suarez, M. F., Gordillo-Rondón, M., Redondo, J., \& Marianela, L. (2017). Estilos de crianza que inciden en la presencia de ciberbullying en un colegio público de Bucaramanga. Revista Psicoespacios, 11(18), 95-114. doi:https:// doi.org/10.25057/issn.2145-2776

Cassiani-Miranda, C., Gómez-Alhach, J., Cubides-Munévar, A., \& Hernández Carrillo, M. (2014). Prevalencia de bullying y factores relacionados en estudiantes de bachillerato de una institución educativa de Cali, Colombia, 2011. Revista de Salud Publica, 16(1), 14-26 doi: https://doi.org/10.15446/ rsap.v16n1.43490

Cepeda-Cuervo, E., Pacheco-Durán, P., García-Barco, L., \& Piraquive-Peña, C. (2008). Acoso Escolar a Estudiantes de Educación Básica y Media Revista de Salud Pública, 10(4), 517-528. Obtenido de https://www.scielosp.org/pdf/ rsap/2008.v10n4/517-528/es

Cuevas, M. C., Hoyos P. A., y Ortiz, Y. (2009). Prevalencia de intimidación en dos instituciones educativas del departamento del Valle del Cauca 2009 Pensamiento Psicológico, 6(13), 153-172. http://revistas.javerianacali.edu.co/ index.php/pensamientopsicologico/article/view/123

De Castro, E., Leite, A., Nascimiento, W. W., Nunes, F. A., \& De Sales, F. (2018). Bullying: Prevalence and factors associated with vitimization and aggression in the school quotidian. Texto \& Contexto-Enfermagem, 27(1), e5500016. http://dx.doi.org/10.1590/0104-07072018005500016 Descargado de http://www.scielo.br/scielo.php?script=sci_arttext\&pid=S0104$07072018000100304 \&$ lng $=p t \& t$ ling $=p t$

Del Rey, R., \& Ortega, R. (2008). Bullying en los países pobres: prevalencia y coexistencia con otras formas de violencia. International Journal of Psychology and Psychological Therapy, 8(1), 39-50. doi: http://hdl.handle. net/11441/59367

Del Río, J., Bringue, X., Sádaba, C., \& González, D. (2009). Cyberbullying: un análisis comparativo en estuユdiantes de Argentina, Brasil, Chile, Colombia, México, Perú y Venezuela. V Congrés Internacional Comunicació I Realitat (págs. 307-315). Barcelona.: Departamento de Comunicación Audiovisual y Publicidad y Literatura. Obtenido de https://dadun.unav.edu/ bitstream/10171/17800/1/articulo-cyberbullying.pdf

Egüez, G. D., y Schulmeyer, M. (2014). Bullying en el departamento de Santa Cruz. Santa Cruz: Bolivia. Gobierno Autónomo Departamental Santa Cruz 1-8. Descargado de https://files.upsa.edu.bo/publicaciones/Revistabullyng.pdf

Erazo, Ó. A. (2016). Identificación y descripción de la intimidación escolar en instituciones educativas del municipio de Popayán . Revista Diversitas: Perspectivas en Psicología, 12(1), 55-72. doi:http://dx.doi.org/10.15332/s17949998.2016.0001.04

Finkelhor, D., Mitchell, K., \& Wolak, J. (2000). Online victimization: A report on the nation's youth. Alexandria, VA.: National Center for Missing and Exploited Children. Obtenido de http://unh.edu/ccrc/pdf/CV138.pdf

Flores, K. (2009). Por el derecho a una vida sin violencia. La Paz-Bolivia: Soipa. Obtenido de https://www. monografias.com/trabajos-pdf4/derecho-vidaescolar-sin-violencia/derecho-vida-escolar-sin-violencia.pdf
Forlim, B., Stelko-Pereira, A. C., \& Williams, L. (2014). Relação entre bullying e sintomas depressivos em estudantes do ensino fundamental [Associations between bullying and depressive]. Revista estudos de Psicologia I Campinas, 31(3), 367-376. Obtenido de https://www.redalyc.org/ pdf/3953/395335439007.pdf

Garaigordobil, M. (2011). Prevalencia y consecuencias del cyberbullying: una revisión. International Journal of Psychology and Psychological Therapy, 11(2), 233-254. Obtenido de https://www.ijpsy.com/volumen11/num2/295/ prevalencia-y-consecuencias-del-cyberbullying-ES.pdf

Garaigordobil. (2013). Cyberbullying. Screening de acoso entre iguales. Screening del acoso escolar presencial (bullying) y tecnológico (cyberbullying). Madrid: TEA. Obtenido de https://docplayer.es/28100965-M-garaigordobilscreening-de-acoso-entre-iguales-screening-del-acoso-escolar-presencialbullying-y-tecnologico-cyberbullying.html

Garaigordobil, M. (2015). Cyberbullying in adolescents and youth in the Basque Country: Prevalence of cybervictims, cyberaggressors, and cyberobservers . Journal of Youth Studies, 18(5), 569-582. doi:http://dx.doi.org/10.1080/13676 261.2014.992324.

Garaigordobil, M. (2017a). Psychometric properties of the Cyberbullying Test, a screening instrument to measure cybervictimization, cyberaggression, and cyberobservation. Journal of Interpersonal Violence, 32(23), 3556-3576. Obtenido de https://doi.org/10.1177/0886260515600165

Garaigordobil. (2017b). Bullying y cyberbullying: definición, prevalencia, consecuencias y mitos. Barcelona: Fundació per la Universitat Oberta de Catalunya.

Garaigordobil. (2018). Bullying y Cyberbullying: Estrategias de evaluación, prevención e intervención. San Sebastian, España., Barcelona: UOC. Oberta UOC Publishing.

Garaigordobil, M., y Martínez-Valderrey, V. (2018). Technological resources to prevent cyberbullying during adolescence: the cyberprogram 2.0 program and the cooperative cybereduca 2.0 videogame. Frontiers in Psychology, 9:745. https://www.frontiersin.org/articles/10.3389/fpsyg.2018.00745/abstract

Garaigordobil, M., y Oñederra, J. A. (2008). Bullying: Incidence of peer violence in the schools of the Autonomous Community of the Basque Country. Revista Internacional de Psicología y Terapia Psicológica / International Journal of Psychology and Psychological Therapy, 8(1), 51-62.

Garaigordobil, M., \& Oñederra, J. A. (2009). Acoso y violencia escolar en la Comunidad Autónoma del País Vasco. Revista Psicothema, 21(1), 83-89. Obtenido de $h$ ttp://www.psicothema.com/psicothema.asp?id=3599

Garaigordobil, M., \& Oñederra, J. A. (2010). La violencia entre iguales: Revisión teórica y estrategias de intervención. Madrid: Pirámide. Obtenido de https:// www.agapea.com/libros/La-violencia-entre-iguales-revision-teorica-yestrategias-de-intervencion-9788436823486-i.htm

García, L., Orellana, O., Pomalaya, R., Yanac, E., Sotelo, L., Herrera, E., . Fernandini, P. (2010). Cyberbullying en escolares de educación secundaria de Lima Metropolitana. Revista de Investigación en Psicología, 13(2), 83-99. Obtenido de http://revistasinvestigacion.unmsm.edu.pe/index.php/psico/ article/view/3714/2979

García-Maldonado, G., Joffre-Velázquez, V. M., Martínez-Salazar, G., \& LlanesCastillo, A. (2011). Cyberbullying: forma virtual de intimidación escolar. Revista Colombiana de psiquiatría, 40(1), 115-130. Obtenido de http://www. scielo.org.co/pdf/rcp/v40n1/v40n1a10

García-Maldonado, G., Martínez-Salazar, G. J., Saldívar-González, A., SánchezNuncio, R., Martínez-Perales, G., \& Barrientos-Gómez, M. d. (2012). Factores de riesgo y consecuencias del cyberbullying en un grupo de adolescentes: Asociación con bullying tradicional. Revista Boletín Médico del Hospital Infantil de México, 69(6), 463-474. Obtenido de http://www. medigraphic. com/pdfs/bmhim/hi-2012/hi126g.pdf

Gómez, C. A. (2014). Factores asociados a la violencia: revisión y posibilidades de abordaje. Revista Iberoamericana de Psicología: Ciencia y Tecnología,, 7(1), 115-124. Obtenido de https://revistas.iberoamericana.edu.co/index.php/ ripsicologia/article/view/486/451

González, V., Mariaca, J. I., \& Arias., A. (2014). Estudio exploratorio del bullying en Medellín. Revista Pensando Psicología, 10(17), 17-25. doi: http://dx.doi. org/10.16925/pe.v10i17.776

Guaygua, G., \& Castillo, B. (2010). In-Seguridad ciudadana: diagnóstico y plan de prevención de la violencia en las escuelas de El Alto. (1. ed ed.). La Paz; Mexico D.F., La Paz, Bolivia. El Alto : Centro de Promoción de la Mujer Gregoria Apaza. Obtenido de http://www.worldcat.org/title/in-seguridadciudadana-diagnostico-y-plan-de-prevencion-de-la-violencia-en-lasescuelas-de-el-alto/oclc/712638174

Herrera-López, M., Romera, E., \& Ortega-Ruiz, R. (2017). Bullying and cyberbullying 
in Colombia: co-occurrence in adolescent schoolchildren. . Revista Latinoamericana de Psicología , 49(3), 163-172. doi:https://doi.org/10.1016/j. rlp.2016.08.001

Kornblit, A. L., \& Adaszko, D. (2007). Factores vinculados a manifestaciones de violencia en el ámbito de la escuela media. Espacios en Blanco. Revista de Educación, 17(1), 137-174. . Obtenido de http://www.redalyc.org/ pdf/3845/384539799006.pdf

Lanzillotti, A., \& Korman, G. (2014). Cyberbullying, características y repercusiones de una nueva modalidad de maltrato escolar. Revista Acta Psiquiátrica y Psicológica de América Latina, 60(1), 36-42. Obtenido de http://ri.conicet. gov.ar/handle/11336/34227

López-Bañuelos, A., Álvarez-Noriega, A., Villalpando-Aguilar, B., Torres-Vera, J., Guzmán-Pérez, J. M., \& Castillo-Sotelo, V. (2012). Diagnóstico de prevalencia del acoso escolar (bullying) en primarias, secundarias y preparatorias del municipio de Tijuana. Tijuana, Mexico. CETYS Universidad. Obtenido de https://www.quao.org/sites/default/files/buenas\%20practicas/ Diagn\%C3\%B3stico\%20de\%20prevalencia\%20del\%20acoso\%20escolar\%20 en\%20primarias\%2C\%20secundarias\%20y\%20preparatorias.pdf

Lucio López, L. A. (2009). Agresores escolares en el ciberespacio el cyberbullying en preparatorias mexicanas del 22-25 de septiembre. XI Asamblea general de ALAFEC, (págs. 1-16). Guayaquil. Obtenido de http://www.academia. edu/4632490/Agresores escolares_en el ciberespacio el cyberbullying en preparatorias mexicanas

Machimbarrena, J. M., \& Garaigordobil, M. (2017). Bullying/Cyberbullying en quinto y sexto curso de primaria: diferencias entre centros públicos y privados. Revista Anales de Psicología , 32(2), 319-326. doi:http://dx.doi.org/10.6018/ analesps.33.2.249381

Mallmann, C. L., Lisboa, C. S., \& Calza, T. Z. (2018). Cyberbullying e estratégias de coping em adolescentes do sul do Brasil. Revista Acta Colombiana de Psicología, 21(1), 23-33. doi: http://www.dx.doi.org/10.14718/ACP.2018.21.1.2

Mendoza, B., Rojas, C., \& Barrera, A. (2017). Rol de participación en bullying y su relación con la ansiedad. Revista Perfiles educativos, 39(158), 38-51. Obtenido de http://www.scielo.org.mx/pdf/peredu/v39n158/0185-2698peredu-39-158-00038.pdt

Morcillo, C., Ramos-Olazagasti, M., Blanco, C., Sala, R., Canino, G., Bird, H., \& Duarte, C. (2015). Socio-Cultural context and bulling others in childhood. Journal of Child and Family Studies, 24(8), 2241-2249. doi: http://doi. org//10.1007/s10826-014-002

Olweus, D. (1999). Norway. En P. K. Smith, Y. Morita, J. Junger-Tas, D. Olweus, R. Catalano y P. Slee (Eds.), The nature of school bullying. A cross-national perspective (pp. 28-48). London: Routledge.

Olweus, D. (1973). Hackkycklingar och oversittare: forsking orn skol-mobbning Estocolmo: Almqvist \& Wiksell. Obtenido de http://www.worldcat.org/title/ hackkycklingar-och-oversittare-forskning-om-skolmobbning/oclc/60927775

Olweus, D. (1993). Bullying at school Whatweknowand: What we can do. Oxford: UK. Blackwell. Obtenido de https://www.amazon.com/Bullying-School-What Know-Can/dp/0631192417/ref=mt_paperback?_encoding=UTF8\&me=\&qid=

Olweus, D. (2013). School Bullying: Development and some important challenges Journal Annual Review of Clinical Psychology, 9(1), 751-780. doi:https://doi. org/10.1146/annurev-clinpsy-050212-185516

Ortega, R., Sánchez, V., Ortega-Rivera, J., Del Rey, R., \& Genebat, R. (2005). Violencia escolar en Nicaragua. Un estudio descriptivo en escuelas de primaria. Revista Mexicana de Investigación Educativa, 10(26), 787-804 Obtenido de https://idus.us.es/xmlui/bitstream/handle/11441/23195/ violencia\%20escolar\%20en\%20nicaragua.pdf?sequence=2

Ponce, Z., \& Cucunubá, C. E. (2014). Bullying escolar y disfunción familiar en estudiantes de $4^{\circ}$ y $5^{\circ}$ grado de educación básica primaria de dos núcleos educativos del distrito de Santa Marta, Colombia. . Revista Psicoespacios, 8(12), 344-377. doi:https://doi.org/10.25057/21452776.304

Prodócimo, E., Cerezo, F., \& Arense, J. J. (2014). Acoso escolar: Variables sociofamiliares como factores de riesgo o de protección. . Revista Psicología Conductual / Behavioral Psychology, 22(2), 343-357. Obtenido de https://www.researchgate.net/profile/Fuensanta_Cerezo/ publication/272085860 Acoso escolar Variables sociofamiiares como factores de riesgo o de proteccion/links/55682c1108aeab77721ebba7/ Acoso-escolar-Variables-sociofamiiares-como-factores-de-riesgo-o-de-p

Redondo, J., Luzardo-Briceño, M., García-Lizarazo, K., \& Inglés, C. (2017). Impacto psicológico del ciberbullying en estudiantes universitarios: un estudio exploratorio. . Revista Colombiana de Ciencias Sociales, 8(2), 458-478. doi:DOI: http://dx.doi.org/10.21501/22161201.2061

Resett, S., \& Gámez-Guadix, M. (2017). Traditional bullying and cyberbullying: differences in emotional problems, and personality. Are cyberbullies more Machiavellians? . Journal of Adolescence, 61, 113-116. doi:https://doi. org/10.1016/j.adolescence.2017.09.013

Resset, S. (2015). Bullying, nominaciones de pares y correlatos psicológicos en adolescentes de escuelas medias. Revista de Psicología, 28, 95-114.

Robalino, G. (2015). Factores que influyen en la prevalencia de bullying en estudiantes de los colegios rurales del cantón Cuenca, Azuay, 2014. Revista de la Facultad de Ciencias Médicas de la Universidad de Cuenca, 33(2), 3747. doi:http://dspace.ucuenca.edu.ec/handle/123456789/25077

Rodríguez-Álvarez, A., \& Delgado de Briceño, G. (2010). Estudio de expresiones de violencia escolar entre estudiantes de escuelas básicas venezolanas. Revista de Investigación, 34(70), 57-69. Obtenido de http://www.scielo.org. ve/scielo.php?script=sci arttext\&pid=\$1010-29142010000200006

Romera, E., Del Rey, R., \& Ortega, R. (2011). Prevalencia y aspectos diferenciales relativos al género del fenómeno bullying en países pobres. Journal Psicothema , 23(4), 624-629. Obtenido de http://www.psicothema.com/ psicothema.asp?id=3932

Romo, M., \& Kelvin, E. (2016). Impact of bullying victimization on suicide and negative health behaviors among adolescents in Latin America. Revista Panamericana de Salud Publica/Pan American Journal of Public Health, 40(5), 347-355. Obtenido de https://www.scielosp.org/article/rpsp/2016. v40n5/347-355/

Santoyo, D., \& Frías, S. (2014). Acoso escolar en México: actores involucrados y sus características. Revista Latinoamericana de Estudios Educativos (México), 44(4), 13-41. . Obtenido de http://hdl.handle.net/123456789/3502

Shephard, B., Ordóñez, M., \& Mora, C. (2015). Estudio Descriptivo: Programa de Prevención y Disminución del Acoso Escolar - "Bullying". Fase Diagnóstica: Prevalencia. Revista Médica HJCA, 7(2), 155-161. Obtenido de https:// publons.com/review/142031/

Silva, K., Andrade, A., Leichsenring, F., \& Hirle, V. (2014). El bullying en los alumnos de quinto año de las escuelas municipales del Reconcavo de Bahia. Revista de Investigación Universitaria,, 3(1), 43-52. Obtenido de http://docplayer. es/50256376-El-bullying-en-los-alumnos-de-quinto-ano-de-las-escuelasmunicipales-del-reconcavo-de-bahia.html

Silva-Villarreal, S., Castillo, S., Eskildsen, E., Vidal, P., Mitre, J., \& Quintero, J. (2013) Prevalencia de bullying en estudiantes de los ciclos básicos y preclínicos de la carrera de medicina de la Universidad de Panamá. Revista Archivos de Medicina, 9(4), 1-8. doi: 10.3823/1205

Uribe, A. F., Orcasita, L. T., \& Aguillón, E. (2012). Bullying, redes de apoyo social y funcionamiento familiar en adolescentes de una institución educativa de Santander, Colombia. Psychologia. Revista Avances de La Disciplina , 6(2), 83-99. Obtenido de http://www.scielo.org.co/pdf/psych/v6n2/v6n2a08.pdf

Valadez, I., Amezcua, R., González, N., Montes, R., \& Vargas, V. (2011). Maltrato entre iguales e intento suicida en sujetos adolescentes escolarizados. Revista Latinoamericana de Ciencias Sociales, Niñez y Juventud, 9(2), 783-796. Obtenido de https://dialnet.unirioja.es/servlet/articulo?codigo=3750956

Varela, J., Pérez, C., Schwaderer, H., Astudillo, J., \& Lecannelier, F. (2014). Caracterización de cyberbullying en el gran Santiago de Chile, en el año 2010. Revista Psicología Escolar E Educacional , 18(2), 347-354. doi:http:// dx.doi.org/10.1590/

Vega-López, M. G., \& González-Pérez, G. J. (2016). Bullying en la escuela secundaria. Revista Mexicana de Investigación Educativa, 21(71), 1165-1189. Obtenido de https://search.proquest.com/ docview/1927956163? accountid $=17248$

Vega-López, M. G., González-Pérez, G. J., \& Quintero-Vega, P. P. (2013) Ciberacoso: Victimización de alumnos en escuelas secundarias públicas de Tlaquepaque, Jalisco, México. Revista de Educación y Desarrollo, 13-20. Obtenido de http://www.cucs.udg.mx/revistas/edu_desarrollo/ anteriores/25/025 Vega.pdf

Vega-López, M. G., González-Pérez, G. J., Valle-Barbosa, M. A., Flores-Villavicencio, M. E., \& Vega-López, A. (2013). Acoso escolar en la zona metropolitana de Guadalajara, México: prevalencia y factores asociados. Revista Salud Colectiva, 9(2), 183-94. Obtenido de https://www.scielosp.org/scielo. php?pid=S1851-82652013000200005\&script=sci arttext

Zalba, J., Durán, L., Carletti, D., Zavala, P., Serralunga, M. G., Jouglard, E. F., \& Esandi, M. E. (2018). Student's perception of school bullying and its impact on academic performance: a longitudinal look. Revista Archivos Argentinos de Pediatría, 116(2), 216-226. Obtenido de https://www.researchgate.net/ profile/Lucas Duran/publication/323425025 Student\%27s perception of school bullying and its impact on academic performance A longitudinal look/links/5a955bdf45851535bcdb71f2/Students-perception-ofschool-bullying-and-its-impac 
